\title{
Thermally activated magnetization reversal in monoatomic magnetic chains on surfaces studied by classical atomistic spin-dynamics simulations
}

\author{
David S. G. Bauer, ${ }^{1}$ Phivos Mavropoulos, ${ }^{1}$, $*$ Samir Lounis, ${ }^{2}$ and Stefan Blügel ${ }^{1}$ \\ ${ }^{1}$ Institut für Festkörperforschung, Institute for Advanced Simulation, \\ and JARA, Forschungszentrum Jülich, D-52425 Jülich, Germany \\ ${ }^{2}$ Department of Physics and Astronomy, University of California Irvine, California 92697, USA
}

(Dated: June 24, 2018)

\begin{abstract}
We analyze the spontaneous magnetization reversal of supported monoatomic chains of finite length due to thermal fluctuations via atomistic spin-dynamics simulations. Our approach is based on the integration of the Landau-Lifshitz equation of motion of a classical spin Hamiltonian at the presence of stochastic forces. The associated magnetization lifetime is found to obey an Arrhenius law with an activation barrier equal to the domain wall energy in the chain. For chains longer than one domain-wall width, the reversal is initiated by nucleation of a reversed magnetization domain primarily at the chain edge followed by a subsequent propagation of the domain wall to the other edge in a random-walk fashion. This results in a linear dependence of the lifetime on the chain length, if the magnetization correlation length is not exceeded. We studied chains of uniaxial and tri-axial anisotropy and found that a tri-axial anisotropy leads to a reduction of the magnetization lifetime due to a higher reversal attempt rate, even though the activation barrier is not changed.
\end{abstract}

PACS numbers:

\section{INTRODUCTION}

Advances in experimental techniques in recent years have made possible the controlled growth and characterization of magnetic chains on non-magnetic crystal surfaces. Such chains can be made as thin as monoatomic, i.e., their cross-section consists of a single atom, while they are usually a few nanometers or tens of nanometers long; they grow, e.g., at terrace step edges, in the "trenches" of (110) surfaces or as inclusions in surface alloys [1-7], so that their structure is rather stable, and they can be studied by a number of spin-sensitive techniques including spin-polarized scanning tunneling microscopy or x-ray magnetic circular dichroism. Magnetic chains, as all magnetic nanostructures, bear technological relevance due to the prospect of miniaturization of magnetic bits for information storage. Particularly appealing, however, in chains is that their geometry is defined by only one parameter, the length, which makes it easier to interpret their physical properties [8].

At such small sizes magnetic states at low-temperature equilibrium consist of a single domain and the possibility of information storage relies solely on the bistability of the magnetization, which is a consequence of magnetocrystalline anisotropy. At low enough temperature the direction of magnetization is trapped close to a local energy minimum for some time before it is reversed by thermal flutuations to another minimum, pointing along the opposite direction. Above some characteristic blocking temperature $T_{\mathrm{B}}$, however, there is a transition to a situation where the magnetization fluctuations are too intense for a stable state to be formed. As the size of

*Electronic address: Ph.Mavropoulos@fz-juelich.de the nanomagnets is below the thermodynamic limit, the transition is not a genuine phase transition but rather a smooth crossover.

These effects are strongly size dependent. In many cases, larger system size leads to higher blocking temperature, reaching the Curie temperature in the thermodynamic limit. However, in two-dimensional [10] and even more in one-dimensional systems, upon increasing the system size $L$, the entropy leads the magnetic state to a multi-domain structure at even low temperatures. The characteristic length, at which this effect sets in, is the magnetization correlation length $\xi(T)$; thus, the effect of magnetic bistability in chains is physically limited by the requirement $L<\xi\left(T_{\mathrm{B}}\right)$.

The temperature-driven magnetization reversal dynamics of nano-particles has been tradidionally studied within the Stoner-Wohlfarth model [11, 12], where the particle magnetization is approximated by a single "macro-spin" of rigid magnitude. However, it is known that excited states departing from the macro-spin approximation can play a significant role even at sizes of a few nanometers [13], while another mechanism sets in, namely reversal through domain nucleation and domain wall propagation 14]; the latter mechanism has been seen also in elongated particles [15], and should be even more relevant in chains [16].

Spin dynamics simulations of magnetic nanostructures are based on a parametrization of the spin-dependent part of the total energy and usually follow one of two paths: either that of a quantum Heisenberg model or the one of a classical Heisenberg model. It is well-known that the quantum model exhausts computational resources at relatively small system sizes, as the Hilbert space increases exponentially with the number of atoms, therefore for system sizes of 50 or 100 atoms only the classical model is practically available. However, there are 
also physical situations where the quantum Heisenberg model is not applicable, in particular magnetic systems on metallic surfaces. The quantum model is based on the assumption of half-integer or integer spins that interact with each other. This is the case with spins on an insulating surface, such as $\mathrm{Mn}$ chains on $\mathrm{CuN}$ [17]. On the other hand, magnetic atoms on metallic surfaces are characterized by non-integer or non-half-integer spin values, which are actually average values of the spin operator on superposition states of the magnetic atoms with the substrate continuous spectrum. In this picture, where substrate electrons hop on and off the magnetic atoms, the spin states are blurred out compared to the sharp eigenstates of the quantum Heisenberg model. In addition, damping of the magnetic excitations into electron-hole pairs emerges, with concequences in g-shift and frequency-dependent magnetization lifetime [18 22]. This, together with temperature effects, causes decoherence of the magnetic states and thus the quantum Heisenberg model is not applicable any more; possibly a classical model, founded on the principles of adiabatic spin dynamics [23], is better suited in this case. Actually the classical atomistic spin dynamics is based on the LandauLifshitz equations of motion, with the point-dependent magnetization being replaced by atom-dependent magnetic moments, and is a widely used tool for the study of atom-dependent nanomagnetism [24, 25].

Having this in mind, we use a classical spin-dynamics simulation method, implemented in our juSpinx code, to study the time evolution of the magnetization in monoatomic chains. Assuming a single-ion anisotropy tensor $\boldsymbol{K}_{i}$, inter-atomic exchange coupling constants $J_{i j}$ between atomic spin moments $\vec{M}_{i}$ and $\vec{M}_{j}$, and an external magnetic field $\vec{B}^{\text {ext }}$, the spin-dependent excitation energy of the system is parametrized by a classical Heisenberg Hamiltonian of the form

$H=-\frac{1}{2} \sum_{i j} J_{i j} \vec{M}_{i} \cdot \vec{M}_{j}-\sum_{i} \vec{M}_{i} \cdot \boldsymbol{K}_{i} \vec{M}_{i}-\sum_{i} \vec{B}^{\text {ext }} \cdot \vec{M}_{i}$

where it is implied that $J_{i i}=0$, while the factor $\frac{1}{2}$ compensates for the double-counting in the summation; $i$ and $j$ denote the atoms in the chain. We set henceforth $\left|\vec{M}_{i}\right|=1$, so that $\vec{M}_{i}$ are to be understood as unit vectors along the atomic moment directions, with the modulus of the moments absorbed in the parameters $J_{i j}, \boldsymbol{K}_{i}$ and $\vec{B}^{\text {ext }}$. Dipole-dipole interactions are neglected here, as we are interested in systems of not more than 100 atoms in size. In order to ensure bistability, the singleion anisotropy should favour a particular axis; the simplest such case is that $\boldsymbol{K}_{i}$ is the same for each atom and its eigenvalues are $\left(K_{x}, K_{y}, K_{z}\right)$, with $K_{x}=K_{y}=0$ and $K_{z}=K>0$, i.e. the anisotropy is uniaxial, so that second term of the Hamiltonian takes the form $-\sum_{i} K M_{i ; z}^{2}$, with the $z$-azis appropriately chosen along the low-energy direction. In the limit $K /\left|J_{i j}\right| \gg 1$, there can be a further simplification, as the system behaviour is well-described by the Ising model and it follows Glauber dynamics [26].
This condition is met e.g. in single chain magnets 27] consisting usually of transition metal atoms that are stabilized and linked in a chain form by molecular ligands. However, for metallic magnetic chains on surfaces the magnetic anisotropy can take large values that are of the order of a few meV, while the exchange coupling is typiclaly one or two orders of magnitude stronger [28]. In this case, an appropriate classical approximation to the spin dynamics is the stochastic Landau-Lifshitz equation of motion:

$$
\hbar \frac{\partial \vec{M}_{i}}{\partial t}=-\vec{M}_{i} \times \vec{B}_{i}^{\mathrm{eff}}-\lambda \vec{M}_{i} \times\left(\vec{M}_{i} \times \vec{B}^{\mathrm{eff}}\right)-\vec{M}_{i} \times \vec{f}_{i}(t)
$$

where we have defined the effective magnetic field acting on the spin at the atomic site $i$ as

$$
\begin{aligned}
\vec{B}_{i}^{\mathrm{eff}} & =-\frac{\partial H}{\partial \vec{M}_{i}} \\
& =\vec{B}^{\mathrm{ext}}+\sum_{j} J_{i j} \vec{M}_{j} \\
& +2\left[K_{i, x}\left(\vec{M}_{i} \cdot \hat{x}\right) \hat{x}+K_{i, y}\left(\vec{M}_{i} \cdot \hat{y}\right) \hat{y}+K_{i, z}\left(\vec{M}_{i} \cdot \hat{z}\right) \hat{z}\right]
\end{aligned}
$$

and $\lambda$ is a damping parameter leading the system to equilibrium at zero temperature. The temperature $T$ enters via a stochastic fluctuating force $\vec{f}_{i}(t)$, as described in Appendix A. In Eq. (2), the first term on the right-hand side is derived directly from the Hamiltonian (11), while the damping and temperature terms are phenomenologically added assuming interaction of the spins with a temperature bath.

Our focus in this work is on the temperature-induced magnetization reversal of finite-length chains. It is implied that the length of the chain, $L$, is considerably smaller than the correlation length $\xi(T)$, otherwise the magnetic configuration consists of many domains, and the bistability loses its meaning. We seek to identify the lifetime $\tau$ of a magnetic state before it is flipped to the opposite magnetization direction. The flip is caused by thermal effects only and we are interested in a "loss of memory", therefore we set $\vec{B}^{\text {ext }}=0$. It is expected, and found in the simulation, that $\tau(T)$ follows to a good approximation an Arrhenius law,

$$
\tau(T)=\tau_{0} \exp \left(E_{\mathrm{B}} / k_{\mathrm{B}} T\right)
$$

where $E_{\mathrm{B}}$ is interpreted as an activation energy barrier and $1 / \tau_{0}$ as an attempt frequency; $k_{\mathrm{B}}$ is the Boltzmann constant (details on the method of calculation of $\tau$ are given in Appendix A). We find that $\tau_{0}$ shows a deviation from a constant only at high temperatures, where the magnetization does not spend any appreciable time at a state before being reversed. What is important, however, is the dependence of $E_{\mathrm{B}}$ and $\tau_{0}$ on the properties of the chain. We keep a minimal model, considering only nearest-neighbour exchange coupling $J=J_{i j}$ and in most cases the same anisotropy $K$ at each site (except when considering edge-anisotropy effects), whence 
the energy- and time-scales of the system are defined by $J, K$, and $\lambda$. Then, a domain wall that is formed in the chain will have a width of approximately $L_{\mathrm{DW}}=2 \sqrt{J / K}$ and a formation energy of $E_{\mathrm{DW}}=2 \sqrt{2 J K}$ (estimated at the continuum limit [29] of Eq. 11). As we explain below, our main findings are categorized in the following regimes: (i) $L \ll L_{\mathrm{DW}}$ : the chain then behaves like a monodomain particle, as laid out already in the theory of Stoner, Wohlfarth and Brown [11, 12], with the reversal being controlled by a barrier of $E_{\mathrm{B}}=L K$. (ii) $L \gg L_{\mathrm{DW}}$ : The magnetization reversal occurs due to a domain nucleation and subsequent domain wall propagation; the barrier entering Eq. (44) is then $E_{\mathrm{B}}=E_{\mathrm{DW}}$. Regime (ii) becomes relevant also for shorter chains as the anisotropy increases, especially above the so-called Ising limit $K / J>1$, when the domain wall becomes atomically sharp; then even very short chains of a few atoms in length behave as in regime (ii). These results are presented in Section II Furthermore, in Section III we investigate the possibility of different anisotropy parameters in all three axes (i.e., we include also an in-plane anisotropy); in this case, entropic arguments imply that the attempt rate $1 / \tau_{0}$ can be strongly affected, and this is verified by our simulations. In Section IV we discuss our findings for realistic system parameters of Co chains on Pt. We conclude with a summary in Section D

\section{CASE OF UNIAXIAL ANISOTROPY}

In the case of surface-supported chains the easy axis of magnetization, driven by spin-orbit coupling, is either perpendicular to the chain or along the chain axis, as can be shown by symmetry arguments 30, 31]. For instance, in the case of Co chains on Pt 1] or Co islands and nanoclusters on Pt [32, 33] the easy axis is perpendicular to the surface (except in the case of chains at step edges, where there is a canting induced by the step edge environment) [4, 30, 34 36]. The anisotropy barrier is typically between 1 and $10 \mathrm{meV}$, strongly dependent on the cluster size or shape. In this section we assume that the anisotropy tensor possesses rotational symmetry in the plane perpendicular to the easy axis; this particular Ansatz is fulfilled in free-standing chains but is not always valid when the chain is adsorbed on a surface. Thus, its approximate validity is strongly system-dependent and a more general case is investigated in Section [II] As a rule of thumb, it should be more valid for chains that are contained in the first surface layer or for chains of biatomic cross section, compared to chains that are on top of the surface layer, because in the former case the in-plane asymmetry of the electron potential is smaller. Other effects that we neglect here are the possibility of temperature-dependent orientation of the easy axis that has been observed in certain systems [37] and a possible canting of the anisotropy axis for the edge atoms, which has been found experimentally and theoretically $[1,30,34$ 36]. However, at the end of the section we dis-

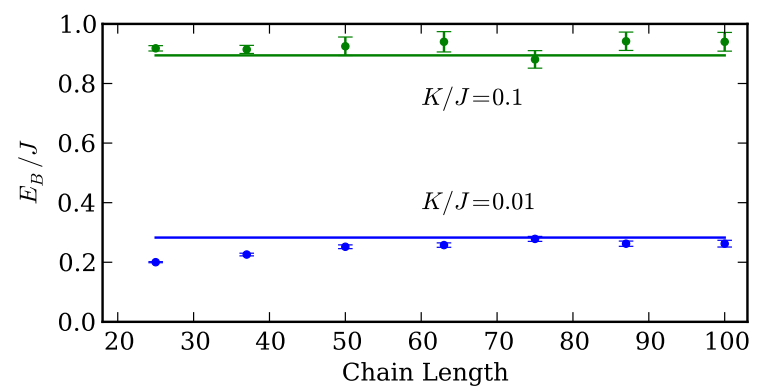

FIG. 1: (colour online) Simulation fits to the activation barrier height as a function of chain length taking into account a uniaxial anisotropy constant $K$. Cases $K / J=0.01$ and $K / J=0.1$ are shown. The horizontal lines correspond to the analytical solution of the continuum approximation $E_{\mathrm{B}} / J=2 \sqrt{2} \sqrt{K / J}$ and agree rather well with the simulation results. For $K / J=0.01$ we see a slight decrease of $E_{\mathrm{B}}$ at small lengths, indicating the onset of a transition to a StonerWohlfarth behaviour as the length becomes comparable to the domain wall width.

cuss some effects that could occur due to increased edge anisotropy.

We proceed with a presentation of our results in the case of uniaxial anisotropy. We simulated the dynamics of monoatomic chains of various lengths up to a maximum of 100 atoms, with $K / J=0.01$ and 0.1 ; we chose a damping of $\lambda=0.1$. Values of $K / J>0.1$ are rather extreme for supported transition-metal chains, but can be realized for example in cases of molecular magnets (where the exchange is comparably week), or for unsupported chains of $4 \mathrm{~d}$ transition metal atoms 38]. The running time of the simulations varied, depending on the system, so that statistically reliable results could be obtained for the lifetime $\tau$.

By fitting the Arrhenius law (44) to our simulation results we extracted $E_{\mathrm{B}}$ and verified the hypothesis that the energy barrier results basically from the formation of a domain wall, if the chain is not too short. The results presented in Fig. 1 1 show that $E_{\mathrm{B}}=E_{\mathrm{DW}}$ holds up to the limit $L \approx L_{\mathrm{DW}}$; for smaller lengths, a deviation is seen (in particular in the case $K / J=0.01$, where $L_{\mathrm{DW}}=20$ ).

Next we present in Fig. 2 simulation data on a chain of 100 spins for $K / J=0.1$ at a temperature of $k_{\mathrm{B}} T / J=$ 0.11 . The abscissa denotes the simulation time broken up in three time intervals; for each interval two panels are shown. In the upper panel, the ordinate shows the chain total magnetic moment along the easy axis ( $z$-axis), $M_{z}^{\text {tot }}$, as a function of time. Reversal events are evident, indicated as $\mathrm{B}, \mathrm{D}$ and $\mathrm{E}$, when the total moment is reversed. Each reversal event is relatively fast, followed by a longer time interval where the total moment is approximately stable at $M_{z}^{\text {tot }}= \pm 85$. However, we also see at $\mathrm{A}, \mathrm{C}$, and $\mathrm{F}$ attempted events that failed, with the total moment returning to its previous state. In the lower panel of Fig. 2 we see a series of snapshots of the system over the same time span, visualizing the time-evolution 


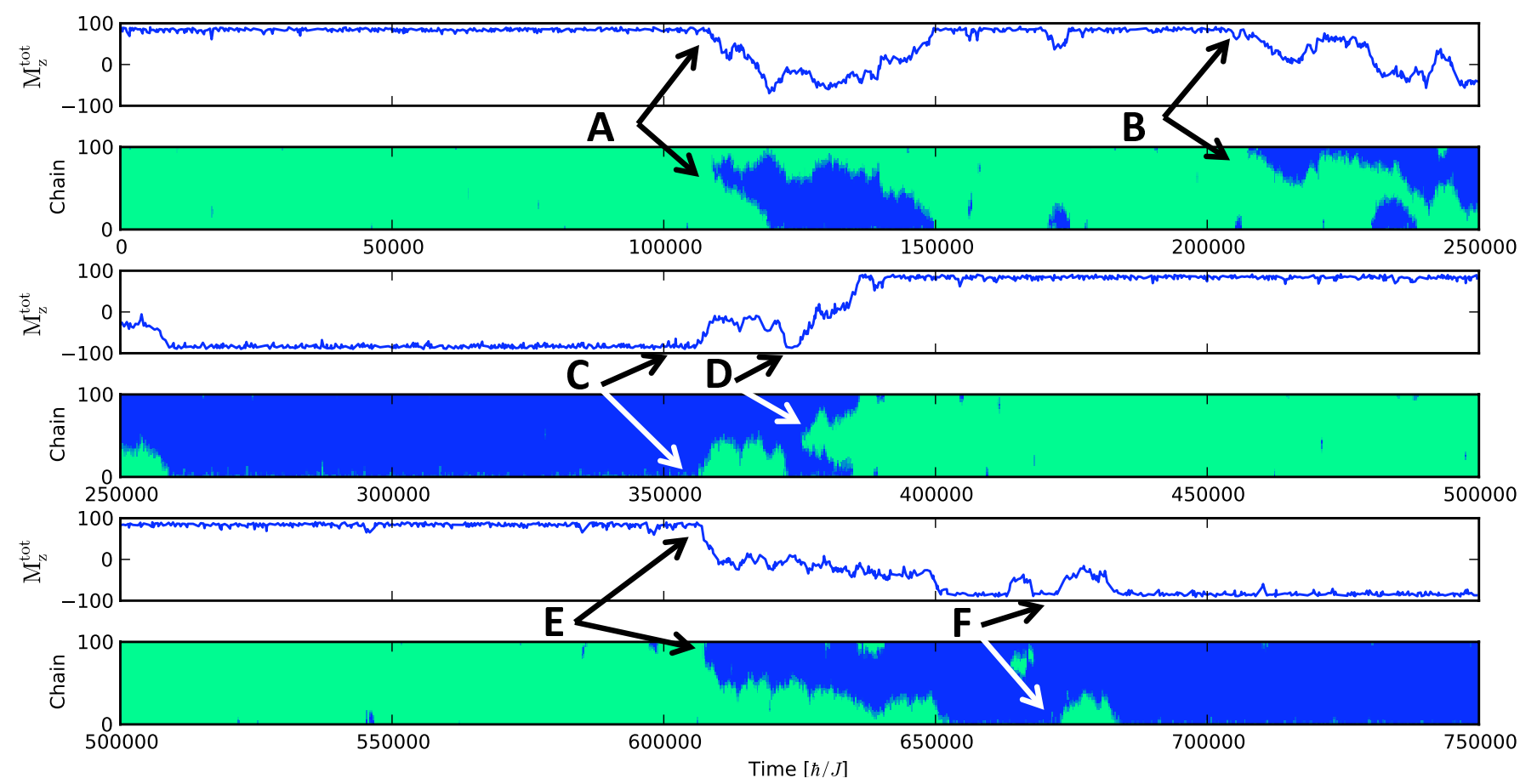

FIG. 2: (colour online) Time evolution of simulation snapshots for a 100 -atom chain with anisotropy $K / J=0.1$. The total simulation time interval is broken up in three sub-intervals of duration $0.25 \times 10^{6} \hbar / J$ each. For each sub-interval, the upper panel shows the $z$-component of the total magnetic moment of the chain, $M_{z}^{\text {tot }}(t)$, while the lower, colour-shaded part shows a snapshot of the $z$-component of the magnetization at any site in time: green for $M_{i ; z}(t)>0$, blue for $M_{i ; z}(t)<0$. In the upper panels the attempts for magnetization reversal are witnessed by a significant change of $M_{z}^{\text {tot }}(t)$, while in the lower panels one can see where the attempt is intitiated and how the magnetization-reversed region evolves in time. The letters and arrows correspond to events that are described in the main part of the text (Sec. II).

of the site-dependent magnetization. Here, the ordinate represents the position of the spin in the chain, $i$, while a colour-code is used for the sign of the local $M_{i ; z}$ : green for $M_{i ; z}>0$, blue for $M_{i ; z}<0$. We can see how reversal attempts start by nucleation of a spin-fliped region that either propagates throughout the chain to end in reversal, as in $\mathrm{B}, \mathrm{D}$, and $\mathrm{E}$, or is annihilated in an unsuccessful attempt as in A, C, and F. We also see that in some attempts the nucleation starts at the chain edge, as in B, C, $\mathrm{E}$ and $\mathrm{F}$, while for others it starts in the chain interior, as in A and D. Although here we show a time-interval containing both kinds of nucleation, at not too high temperatures nucleation is more likely to occur at the edges than in the interior, because in the latter case two domain walls must appear simultaneously requiring the system to overcome a twice as high energy barrier (the latter process is known as soliton-antisoliton creation and has been studied e.g. by Braun [16]). For the same reason it is statistically rare for more than one nucleation regions to be present simultaneously, at least as long as $L \ll \xi$, therefore we consider in our qualitative analysis only single nucleation events at the edges. Our arguments are supported by calculations making use of a transfer-matrix approach to calculate thermodynamic properties of finite anisotropic Heisenberg [4] showing that the edge-atoms of chains are subject to significantly stronger fluctuations than the atoms in the interior.

The reversal time itself, i.e. the time that it takes for the domain wall to propagate through the system once it is created, is in general much shorter than the magneticstate lifetime $\tau$. This effect is also seen experimentally by spin-polarized scanning tunneling microscopy of ferromagnetic nano-islands [15]. There, fast reversal occurs during the scanning process, resulting in an image of seemingly two domains, while in reality the island is in a mono-domain state.

The domain wall that occurs during nucleation performs a random walk back-and-forth in the chain, and eventually either returns to its starting point, resulting in an unsuccesful attempt, or reaches the other side, resulting in reversal. The probability for the latter to happen in a random-walk model is [39] $p(L)=1 / L$. Therefore, at constant temperature, the prefactor $\tau_{0}$ of Eq. (4) rises linearly with $L: \tau_{0}(L)=\alpha L$, at least as long as $L_{\mathrm{DW}} \ll L$; this has been found also in simulations of Ising-model dynamics [40]. More accurately, going beyond the Ising model, we must account for the fact that a domain wall centered at position $x$ is attracted to the chain edge if it is too close, i.e., if $x<\frac{1}{2} L_{\mathrm{DW}}$ or $L-x<\frac{1}{2} L_{\mathrm{DW}}$. Thus the domain wall propagation corresponds to a random walk only between $\frac{1}{2} L_{\mathrm{DW}}$ and $L-\frac{1}{2} L_{\mathrm{DW}}$, so that we should 


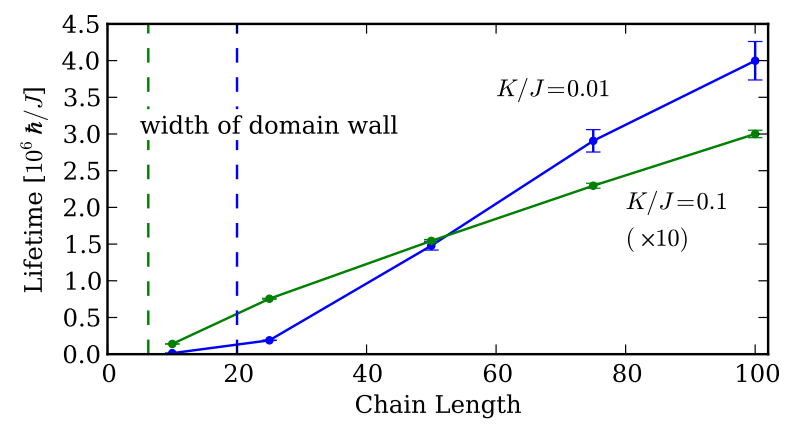

FIG. 3: (colour online) Magnetization lifetime $\tau$ as a function of chain length $L$ for the cases $K / J=0.01$ and $K / J=0.1$ (the latter was scaled up by a factor 10 for better legibility). The linear increase of $\tau$ with $L$ is due to the random-walk-type propagation of the domain wall after nucleation. The offset of the linear behaviour is due to the finite domain wall width. Vertical lines indicate the domain wall widths in the two cases $\left(L_{\mathrm{DW}}=6\right.$ for $K / J=0.1, L_{\mathrm{DW}}=20$ for $\left.K / J=0.01\right)$.

observe an offset in the linear behaviour:

$$
\tau_{0}(L) \propto\left(L-L_{\mathrm{DW}}\right), \text { for } L>L_{\mathrm{DW}} .
$$

This rule is reproduced by the results of our simulations, as shown in Fig. 3 where the calculated lifetime is plotted against the chain length. The offset in the 1st curve $(K / J=0.01)$ can be seen at lengths smaller or comparable to the domain wall width (up to 20-25 atoms). Then, for $L \approx L_{\mathrm{DW}}$, the switching mechanism is intermediate between nucleation and Stoner-Wohlfarth-like coherent rotation, and a kink in the curve $\tau(L)$ is seen. The offset is much reduced in the 2nd curve of Fig. 3 where $K / J=0.1$, because the increased anisotropy leads to smaller $L_{\mathrm{DW}} \approx 6$. A deviation from the rule (5), in particular a saturation, is expected to begin as the chain length becomes too large approaching the correlation length. Then simultaneous occurence of more that one nucleation becomes statistically more and more relevant, until eventually the space-integrated magnetization vanishes at all times. Note that this linear increase (5) of the lifetime with system size is in marked difference to the behaviour of a Stoner-Wohlfarth-type threedimensional particle, where the system size (number of atoms $n$ ) enters multiplicatively in the Arrhenus barrier, $E_{\mathrm{B}}=n K$, causing an exponential dependence of $\tau$ on $n$. On the other hand, in the regime of validity of Eq. (5) ( $\left.L>L_{\mathrm{DW}}\right)$, the Arrhenius law (4) is still valid, but with $E_{\mathrm{B}}$ a constant (i.e., independent of the number of atoms).

We close this section by an observation concerning the anisotropy of the edge atoms of the chain. It is wellknown that, due to the different environment (only one neighbour) the anisotropy tensor of edge atoms, $\boldsymbol{K}_{\text {edge }}$, can differ from the one in the chain interior, in both the direction of the principal axes and in the anisotropy strength, $K_{\text {edge }}$. These effects can affect the nucleation barrier as well as the nucleation frequency. However, there can be in principle an additional effect. Under "nor- mal" conditions a domain wall close to the edge is further attracted toward the edge and in the end expelled form the chain and annihilated, but if $K_{\text {edge }}$ becomes large enough, it will act as a barrier against the annihilation. Then the domain wall will be trapped in the interior of the chain for a longer time, eventually overcoming the barrier due to thermal fluctuations and becoming annihilated. Naturally, in such a situation the nucleation is also more difficult to achieve. We derived analytically an approximate condition for such an edge barrier by considering the energy as a function of rotation of the edge spin and treating the rest of the chain in the continuum limit (see Appendix B). The condition reads:

$$
K_{\text {edge }}>K+E_{\text {DW }} / 4
$$

and is approximately reproduced by simulations that we performed.

\section{CASE OF TRIAXIAL ANISOTROPY}

As we commented earlier, the magnetic anisotropy of a chain on a surface is never really uniaxial, due to the asymmetry of the environment. We take this into account in the simplest way by introducing three eigenvalues of the anisotropy tensor,

$$
\boldsymbol{K}=\left(\begin{array}{ccc}
K_{x} & 0 & 0 \\
0 & K_{y} & 0 \\
0 & 0 & K_{z}
\end{array}\right)
$$

corresponding so to say to an easy, medium, and hard axis, so that the associated energy expression becomes $-\vec{M} \cdot \boldsymbol{K} \vec{M}=-K_{x} M_{x}^{2}-K_{y} M_{y}^{2}-K_{z} M_{z}^{2}$; the site index $i$ has been suppressed here, and it is assumed that all sites have the same anisotropy. Without loss of generality we fix $K_{z} \geq K_{y} \geq K_{x}=0$ (the latter equality can be adjusted by a shift of the energy zero that does not affect the equations of motion, while the order of the inequalities will not affect the statistical results on the lifetime, merely the path that the spins follow to flip). Thus, in effect the two energy minima of the single-ion anisotropy are along the $\pm z$-axis, separated by a low anisotropy barrier $\Delta_{\text {low }}=\left(K_{z}-K_{y}\right)$ if the spin rotates in the $y$ - $z$-plane or by a high anisotropy barrier $\Delta_{\text {high }}=K_{z}$ if the spin rotates in the $x$ - $z$-plane; effects of the exchange $J$ come on top of this as before.

The question that we seek to answer is how the presence of the second anisotropy barrier affects the magnetization lifetime. To make the question more concise, we wish to compare the lifetime in the previously calculated uniaxial case, where $\Delta_{\text {high }}=\Delta_{\text {low }}=K$, to the case $\Delta_{\text {high }}>\Delta_{\text {low }}=K$. The minimal barrier for domain wall formation in long chains or for coherent rotation in short chains remains $2 \sqrt{2 J K}$ or $L K$ respectively, so that the exponent $E_{\mathrm{B}}$ in the Arrhenius law (4) should not be affected. However, the attempt frequency $\tau_{0}^{-1}$ is expected to change, as the energy lanscape of the paths 
that connect the two minima is now different. For example, in the case of uniaxial anisotropy both Bloch-type and Néel-type walls are equal in energy, while in the triaxial case one of the two types will correspond to a higher energy; this must give a difference in entropy.

As it turns out, for a given $\Delta_{\text {low }}$, the lifetime decreases with increasing $\Delta_{\text {high }}$; our findings are summarized in Fig. 4. This is at a first glance counter-intuitive: the fluctuating force enforces a random walk, regulated by the barriers, that has to climb up from an energy minimum to a maximum or saddle point before descending to the opposite minimum. Increasing $\Delta_{\text {high }}$ means that part of the lanscape is visited with smaller frequency, so that there are less escape paths. Then part of the randomwalk steps would be, so to say, waisted in attempts to climb up the high barrier. However, contrary to the case of a confined particle, the latter argument is not valid in the case of spins, because of the precessional motion. The spin trajectory has a component following the direction of the effective field (toward the energy minimum), given by the damping term of Eq. (2), but the stronger, precessional part drives the spin perpendicular to the energy gradient. Therefore, whenever the fluctuating field forces the spin in the direction of the high barrier, the precession drives it in the direction of the low barrier. In this way, the random-walk steps in the direction of the high barrier are not waisted but converted into random-walk steps in the direction of the low barrier. The shape of the resulting trajectory approximates an ellipsis with its long axis along the low-barrier direction. The random walk becomes in a sense more and more one-dimensional, as the difference $\Delta_{\text {high }}-\Delta_{\text {low }}$ increases, and it is known that random walks in one dimension propagate faster away from the starting point than in two dimensions. Therefore the magnetization lifetime becomes shorter. In the limit $\Delta_{\text {high }} \gg \Delta_{\text {low }}$, the situation resembles that of an $y$-z-easy-plane model with a hard axis in the $x$ direction, which obeys the dynamics of precessional switching, long known in micromagnetics [41]. At this limit, even a small deviation of the spin toward the $x$ direction due to the fluctuating force will cause a lengthy precession around $x$, in the $y-z$ plane, which can even last a few rotations if the damping is very low.

The behaviour is visualized for a single spin in Fig. 5 , On the left panel we see a top-view of the trajectory of a spin under a uniaxial anisotropy, with $K_{x}=K_{y}=0$, $K_{z}=K>0$, starting from the state $M_{x}=M_{y}=0$, $M_{z}=1$. The spin is driven away from equilibrium by a fluctuating field, and the trajectory is, on the average, isotropic in $x$ and $y$. On the right panel we see the trajectory for the same simulation time and under the same conditions, with the exception that here $\Delta_{\text {low }}=K$, $\Delta_{\text {high }}=10 K$. The point is not just that the trajectory is elliptical instead of isotropic, but also that in the same time interval the spin has managed to reach closer to a "turning point" of the barrier, where $M_{x}^{2}+M_{y}^{2}=1$, $M_{z}=0$.

In the case of chains (Fig. 4) we observe in the top
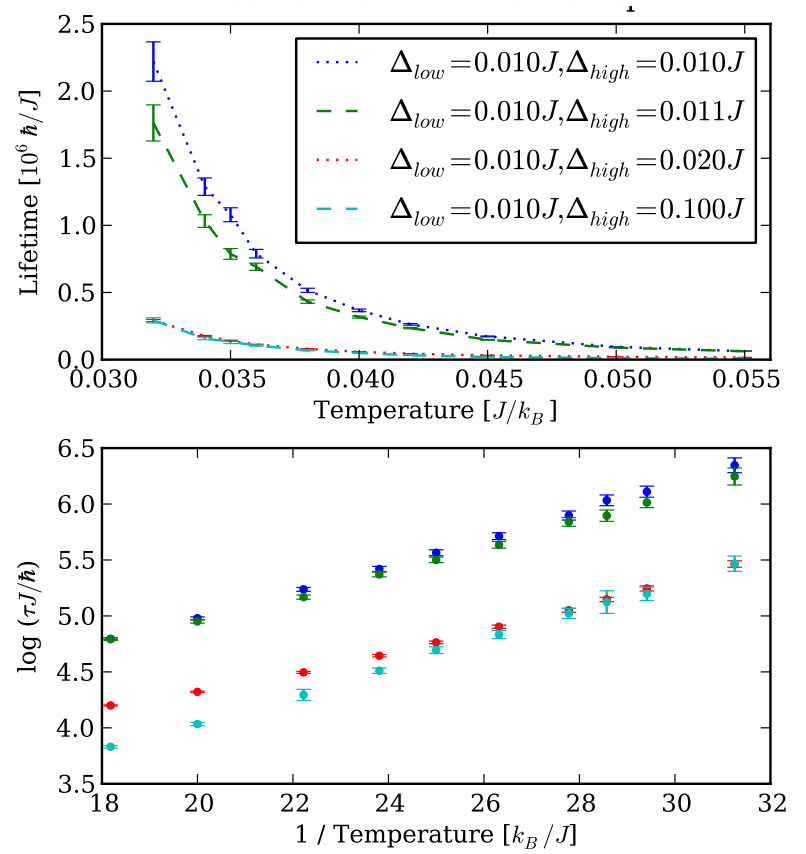

FIG. 4: (colour online) Magnetization lifetime as a function of temperature for a 100-atom chain for several single-ion anisotropy barriers, including triaxial anisotropy (top, linear scale; bottom: logarithm of the lifetime vs. inverse temperature). The low barrier, $\Delta_{\text {low }}=0.01 J$, is kept constant, while $\Delta_{\text {high }}$ is varied between $\Delta_{\text {low }}$ and $10 \Delta_{\text {low }}$. A significant reduction of the lifetime is observed even at a moderate difference of $10 \%$ between $\Delta_{\text {high }}$ and $\Delta_{\text {low }}$. At very high difference a saturation is seen. The activation barrier is in all cases the same, equal to the domain wall energy, $E_{\mathrm{B}}=2 \sqrt{2 J \Delta_{\text {low }}}$, as can be seen in the lower panel where all data sets show the same slope.

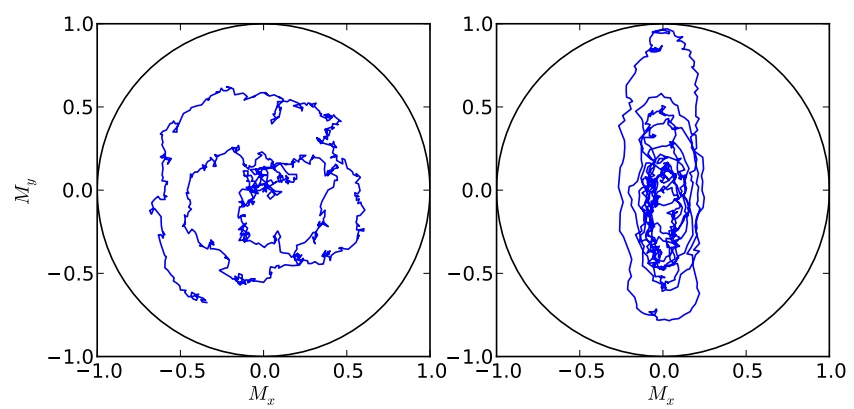

FIG. 5: (colour online) Trajectory of a single spin under anisotropy and fluctuating force. Left: Uniaxial anisotropy, $K_{x}=K_{y}=0, K_{z}=K>0\left(\Delta_{\text {high }}=\Delta_{\text {low }}=K\right)$. Right: $K_{x}=0, K_{y}=9 K, K_{z}=10 K\left(\Delta_{\text {high }}=10 K, \Delta_{\text {low }}=K\right)$. Both simulations correspond to the same total simulation time starting from the state $M_{x}=M_{y}=0$. Evidently, in the presence of different barriers in $x$ and $y$ directions (right panel) the spin reaches faster the turning point at the edge of the circle $\left(M_{x}^{2}+M_{y}^{2}=1, M_{z}=0\right)$. 
panel a drop of the lifetime with increasing $\Delta_{\text {high }}$, while keeping $\Delta_{\text {low }}=0.01 J$ fixed. The curves show the lifetime as a function of temperature. At all temperatures, the longest lifetime is observed for $\Delta_{\text {high }}=\Delta_{\text {low }}$, and it is evident that even a small increase of $10 \%$ in $\Delta_{\text {high }}$ can affect the lifetime quite noticeably. When $\Delta_{\text {high }}$ is twice the value of $\Delta_{\text {low }}$, the lifetime is reduced by an order of magnitude. However there appears also to be a saturation: further increase of $\Delta_{\text {high }}$ to nine times the value of $\Delta_{\text {low }}$ makes no significant difference any more for the lifetime.

In the lower panel, where the same simulation data are shown in a $\log \tau v s .1 / T$ plot, the Arrhenius behaviour as well as the dependence on $\Delta_{\text {high }}-\Delta_{\text {low }}$ becomes easier to see also at higher temperatures; evidently the activation barrier is the same in all cases (and equal to $2 \sqrt{2 J \Delta_{\text {low }}}$ ), as can be seen from the fact that the slope of $\log \tau v s .1 / T$ does not change. Note that at low temperatures the data have larger error bars due to the fewer switches that occur in a given simulation time interval.

\section{REALISTIC PARAMETERS AND RESULTS FOR Co CHAINS ON Pt}

Now we relate our general analysis to realistic systems. For the typical system of supported monoatomic Co chains and nanoislands, for example, values of $J$ of the order of 20-60 meV on $\mathrm{Cu}, \mathrm{Au}$ and $\mathrm{Pt}$ surfaces have been calculated and reported in the literature [9, 42 44], depending on structural parameters such as island or chain geometry, size and substrate. Similar is the case with Fe nanostructures. The anisotropy, on the other hand, is found in experiments to vary by two orders of magnitude depending on structural parameters [32], from the sub-meV range for large clusters up to values as high as $10 \mathrm{meV}$ for single adatoms; for monoatomic Co chains on $\mathrm{Pt}$ (997) step edges, a value of $2 \mathrm{meV}$ has been found by experiments [1], while the value drops to $0.34 \mathrm{meV}$ for biatomic chains. Clearly, the anisotropy is much more sensitive than the exchange coupling. Concerning the appearance of two anisotropy barriers, $\Delta_{\text {low }}$ and $\Delta_{\text {high }}$, calculations [45] on Co monoatomic chains on $\mathrm{Pt}(111)$ yield $\Delta_{\text {low }}=0.8 \mathrm{meV}$ and $\Delta_{\text {high }}=1 \mathrm{meV}$; this $25 \%$ difference between $\Delta_{\text {low }}$ and $\Delta_{\text {high }}$ can play a significant role on the lifetime, as our calculations of Sec. III show.

Following the above observations we are in a position to make an order-of-magnitude prediction for the lifetime of Co monoatomic chains on $\mathrm{Pt}$. We assume values of $J=50 \mathrm{meV}, \Delta_{\text {low }} / J=0.016 \Delta_{\text {high }} / J=0.02$. The only parameter that is at this point arbitrary is the damping, here chosen to be $\lambda=0.1$, which is not actually known for the case of Co monoatomic chains on Pt. In permalloy, for example, the damping is one order of magnitude smaller [46]. However, $\mathrm{Pt}$ is characterized by a much stronger spin-orbit coupling, which can enhance the damping (for example, FePt nanoparticles are reported to have a damping parameter as high as 0.76 [47]). Our current choice has to be corrected once there is more experimental or theoretical data, including exchange parameters beyond first neighbours and an account of the anisotropic exchange (DzyaloshinskiiMoriya interaction), on the particular type of systems. Keeping this in mind, we find for a 50-atom chain an inverse attempt rate of $\tau_{0} \approx 0.6 \mathrm{ps}$ and a barrier of $E_{\mathrm{B}}=2 \sqrt{2 J \Delta_{\text {low }}} \approx 18 \mathrm{meV}$ to be substituted in the Arrhenius law (4). Assuming detection of the reversal by a scanning tunneling microscope (see e.g. Refs. 15] and [48]), and given that this method has a relatively low time resolution of the order of msec [49], we consider a lifetime of 1 second as reasonable for a reliable experimental analysis of spontaneous reversal. Then our simulation suggests that experiments on 50-atom-long chains require temperatures of approximately 10 Kelvin or lower.

\section{CONCLUSIONS}

We have performed classical simulations of atomistic spin dynamics for supported monoatomic magnetic chains, with focus on the magnetization lifetime between temperature-induced subsequent magnetization reversals at chain lengths below the correlation length. The calculations were carried out by integrating the LandauLifshitz equation of motion of classical spin systems in the presence of stochastic forces.

In summary, the main findings of this work are the following. (i) For long chains (longer than the width of a domain wall), the lifetime is governed by an Arrhenius law (44), with a barrier equal to the domain wall energy. For short chains, the barrier eventually becomes equal to the anisotropy energy. (ii) For long chains, the mechanism for reversal is governed by nucleation of a region of reversed magnetization at the chain boundary, forming a domain wall, and propagation of the domain wall through the chain via a random-walk-like procedure. This yields a linear dependence of the lifetime on the chain length for chains longer than the domain-wall width, contrary to an exponential dependence that would be expected for a Stoner-Wohlfarth type of system. (iii) In case of a triaxial anisotropy tensor with non-degenerate eigenvalues, i.e., with a low and a high anisotropy barrier for magnetization reversal, the lifetime is reduced compared to the case of only a low barrier. This effect is related to the precessional motion of the spin, which tends to transform fluctuations in the high-barrier direction into fluctuations in the low-barrier direction. A triaxial anisotropy should always be present in surface-supported chains, as can be argued on the grounds of symmetry of the system.

As far as fundamental science is concerned, the most important question to be answered by this type of calculations is perhaps the one of the limitations in size, time and temperature, that the classical spin dynamics is still a valid approximation, especially in metallic systems. In principle, all ingredients that enter the Landau-Lifshitz equations are experimentally accessible; even more so for 
chains, where the geometry is simple enough so as not to introduce further arbitrariness. The result of the simulations, in particular the magnetization lifetime, is also accessible by experiment. A systematic study in this direction will be highly interesting.

\section{Acknowledgements}

We are greatful to Dr. Riccardo Hertel for discussions on the physics of magnetization dynamics, Dr. Laszlo Szunyogh for discussions on the form of the anisotropy tensor in surface-supported chains, and Prof. Christian Schröder for discussions on the methodology of atomistic spin dynamics. This work has been supported in part by FP7 EU-ITN FANTOMAS. S. L. wishes to thank the Alexander von Humboldt Foundation for a Feodor Lynen Fellowship and also Prof. D. L. Mills for hospitality at the UC-Irvine.

\section{Appendix A: Some details on the method of calculation}

Equation (2) is a stochastic differential equation with multiplicative noise which is interpreted in the Stratonovich sense. The fluctuating force $\vec{f}_{i}(t)$ has white-noise properties, i.e. (i) its time-average vanishes: $\left\langle\vec{f}_{i}\right\rangle=0 ;$ (ii) it is fluctuating much faster than the characteristic precession time so that timeseparated and space-separated fluctuations are decorrelated: $\left\langle\left[\vec{f}_{i}(t)\right]_{\alpha}\left[\vec{f}_{j}\left(t^{\prime}\right)\right]_{\beta}\right\rangle=\delta_{i j} \delta_{\alpha \beta} \delta\left(t-t^{\prime}\right)$, where $\alpha, \beta=$ $x, y, z$ denote the vector components and $i, j$ are atomic sites; and (iii) its amplitude $\epsilon$ is connected to the damping via the fluctuation-dissipation theorem: $\epsilon^{2}=2 \lambda k_{\mathrm{B}} T$ with $k_{\mathrm{B}}$ the Boltzmann constant. Eq. (2) is integrated via a weak Runge-Kutta method which was suggested by Milstein and Tretyakov [50]. The error scales with the step size as $\mathcal{O}\left(h^{4}+\epsilon^{2} h^{2}\right)$. Further information can be found in Ref. [24].

In practice, at each Runge-Kutta step and for each spin at site $i$, a fluctuating field $\vec{f}_{i}$ is calculated by a set of random numbers obeying an appropriate distribution so that the above requirements (i-iii) are fulfilled. Requirement (iii), in particular, guarantees that the fluctuating field intensity corresponds to the particular simulation temperature $T$. The field $\overrightarrow{f_{i}}$ acts in addition to the effective field $\vec{B}_{i}^{\text {eff }}$ and to the damping term as shown in Eq. (2). The random nature of $\overrightarrow{f_{i}}$ comes into notice in the noise observed in the time-dependent magnetiation in Fig. 2. For the calculation of $\tau(T)$, during a sufficiently long simulation time at a fixed temperature, successive time intervals $\Delta t_{i}$ between $N$ successive reversal events (as the ones seen in Fig. 2) are recorded, and at the end averaged as $\tau(T)=\sum_{i=1}^{N} \Delta t_{i} / N$.

\section{Appendix B: Justification of Expression (6)}

Consider a domain wall where the magnetic moment of each atom $i$ has an angle $\theta_{i}$ with respect to the easy axis (the easy axis is taken to be the same for all atoms). Suppose that the wall is not at the interior of the chain, but close to the edge, and that it is formed by constraining the moment of the first atom (labelled " 0 ") by $\theta_{0}$; we accept the boundary condition at the deep interior of the chain that $\theta_{i}=0$ for $i \rightarrow \infty$. Then, a boundary condition at the edge $\theta_{0}=\pi$ corresponds to a fully formed domain wall, while $\theta_{0}=0$ corresponds to the ferromagnetic ground state.

Now we take a fixed $\theta_{0}, 0<\theta_{0}<\pi$. The resulting structure will have the form of a domain wall that has been abruptly cut at $i=0$. If we assume in the discrete model a nearest neighbour distance of length $a$ and employ the continuum approximation [29], then the site index $i$ changes to a continuous variable $x / a$ and we obtain an explicit energy functional for a chain starting with a restricted angle of $\theta_{0}$ at $x=0$ :

$$
E\left(\theta_{0}\right)=\int_{0}^{\infty} d x\left(\frac{1}{2} J a\left(\frac{d \theta}{d x}\right)^{2}+\frac{K}{a} \sin ^{2}(\theta(x))\right) .
$$

Minimizing the energy functional under the boundary conditions $\theta(0)=\theta_{0}$ and $\theta(\infty)=0$ leads to an energy of the domain wall of

$$
E_{\mathrm{DW}}\left(\theta_{0}\right)=\sqrt{2 J K}\left(1-\cos \theta_{0}\right),
$$

which will be used as an approximation to the discrete model.

Next we increase the anisotropy of only the edge atom from $K$ to $K_{\text {edge }}$. This change affects only the energy of the edge atom, changing it by $-K_{\text {edge }} \cos ^{2} \theta_{0}+K \cos ^{2} \theta_{0}$, while the configuration remains the same. We have now an energy

$$
E\left(\theta_{0}\right)=\sqrt{2 J K}\left(1-\cos \theta_{0}\right)-K_{\text {edge }} \cos ^{2} \theta_{0}+K \cos ^{2} \theta_{0} .
$$

For the domain wall to be trapped in the interior of the chain, this expression must have a maximum for $0<\theta_{0}<$ $\pi$, corresponding to the high-point of an energy barrier upon rotation of the edge spin. Differentiation of (B3) with respect to $\theta_{0}$ shows that such a maximum exists under the condition

$$
K_{\text {edge }}-K>\sqrt{J K / 2},
$$

which is equivalent to Expression (6).
[1] P. Gambardella, A. Dallmeyer, K. Maiti, M.C. Malagoli, W. Eberhardt,, K. Kern, and C. Carbone, Nature (Lon- don) 416, 301 (2002). 
[2] L. Hammer, W. Meier, A. Schmidt, and K. Heinz, Phys. Rev. B 67, 125422 (2003).

[3] L. Yan, M. Przybylski, Y. Lu, W.H. Wang, J. Barthel, and J. Kirschner, Appl. Phys. Lett. 86, 102503 (2005).

[4] A. Vindigni, A. Rettori, M.G. Pini, C. Carbone, and P. Gambardella, Appl. Phys. A 82, 385 (2006).

[5] D. Serrate, P. Ferriani, Y. Yoshida, S.-W. Hla, M. Menzel, K. von Bergmann, S. Heinze, A. Kubetzka, and R. Wiesendanger, Nature Nanotechnology 5, 350 (2010).

[6] D. H. Wei, C. L. Gao, Kh. Zakeri, and M. Przybylski, Phys. Rev. Lett. 103, 225504 (2009); O. V. Stepanyuk, N. N. Negulyaev, P. A. Ignatiev, M. Przybylski, W. Hergert, A.M. Saletsky, and J. Kirschner, Phys. Rev. B 79 , 155410 (2009).

[7] J. Honolka, T.Y. Lee, K. Kuhnke, A. Enders, R. Skomski, S. Bornemann, S. Mankovsky, J. Minár, J. Staunton, H. Ebert, M. Hessler, K. Fauth, G. Schütz, A. Buchsbaum, M. Schmid, P. Varga, and K. Kern, Phys. Rev. Lett. 102, 067207 (2009)

[8] Contrary to chains, calculations show that in nanoislands and nanoclusters the inter-atomic exchange interactions fluctuate strongly as a function of cluster shape and size and of the position of the atoms in the cluster [9].

[9] P. Mavropoulos, S. Lounis, and S. Blügel, Phys. Status Solidi B 247, 1187 (2010).

[10] N.D. Mermin and H. Wagner, Phys. Rev. Lett. 17, 1133 (1966).

[11] E.C. Stoner and E.P. Wohlfarth, Phil. Trans. Roy. Soc. (London) A240, 599 (1948).

[12] W.F. Brown Jr., Phys. Rev. 130, 1677 (1963).

[13] S. Rohart, P. Campiglio, V. Repain, Y. Nahas, C. Chacon, Y. Girard, J. Lagoute, A. Thiaville, and S. Rousset, Phys. Rev. Lett. 104, 137202 (2010).

[14] P. Gambardella, A. Dallmeyer, K. Maiti, M. C. Malagoli, S. Rusponi, P. Ohresser, W. Eberhardt, C. Carbone, and K. Kern, Phys. Rev. Lett. 93, 077203 (2004).

[15] M. Bode, O. Pietzsch, A. Kubetzka, and R. Wiesendanger, Phys. Rev. Lett. 92, 067201 (2004).

[16] H.-B. Braun, J. Appl. Phys. 99, 08F908 (2006).

[17] C.F. Hirjibehedin, C.P. Lutz, and A.J. Heinrich, Science 312, 1021 (2006).

[18] T. Balashov, T. Schuh, A. F. Takcs, A. Ernst, S. Ostanin, J. Henk, I. Mertig, P. Bruno, T. Miyamachi, S. Suga, and W. Wulfhekel, Phys. Rev. Lett. 102, 257203 (2009).

[19] P. Lederer and D.L. Mills, Phys. Rev. 160, 590 (1967)

[20] R.B. Muniz and D.L. Mills, Phys. Rev. B 68, 224414 (2003).

[21] S. Lounis, A.T. Costa, R.B. Muniz, and D.L. Mills, Phys. Rev. Lett. 105, 187205 (2010); S. Lounis, A.T. Costa, R.B. Muniz, and D.L. Mills, arXiv:1010.1293 (2010).

[22] A.A. Khajetoorians, S. Lounis, B. Chilian, A.T. Costa, L. Zhou, D. L. Mills, J. Wiebe, and R. Wiesendanger, arXiv:1010.1284 (2010).

[23] V. P. Antropov, M. I. Katsnelson, B. N. Harmon, M. van Schilfgaarde, and D. Kusnezov, Phys. Rev. B 54, 1019 (1996).

[24] V.P. Antropov, S.V. Tretyakov, and B.N. Harmon, J. Appl. Phys. 81, 3961 (1997).

[25] B. Skubic, J. Hellsvik, L. Nordström, and O. Eriksson, J. Phys.: Condens. Matter 20, 315203 (2008); Christian Schröder, H.-J. Schmidt, J. Schnack, and M. Luban, Phys. Rev. Lett. 94, 207203 (2005); N. Kazantseva, D. Hinzke, U. Nowak, R.W. Chantrell, and O. ChubykaloFesenko, Phys. Status Sol. (b) 244, 4389 (2007); A.V.
Savin, G.P. Tsironis, and X. Zotos, Phys. Rev. B 75, 214305 (2007); T. Jourdan, A. Marty, and F. Lancon, Phys. Rev. B 77, 224428 (2008).

[26] R.J. Glauber, J. Math. Phys. 4, 294 (1963).

[27] K. Bernot, L. Bogani, A. Caneschi, D. Gatteschi, and R. Sessoli, J. Am. Chem. Soc. 128, 7947 (2006).

[28] Y. Mokrousov, G. Bihlmayer, S. Blügel, and S. Heinze, Phys. Rev. B 75, 104413 (2007).

[29] A. Aharoni, Introduction to the theory of ferromagnetism, 2nd. edition, Oxford University Press (2000).

[30] B. Lazarovits, B. Újfalussy, L. Szunyogh, G.M. Stocks, and P. Weinberger, J. Phys.: Condens. Matter 16, S5833 (2004).

[31] This is strictly true only if we neglect a possible weak variation of the single-ion anisotropy due to the geometric arangement of the nearest neighbours on the substrate. E.g. the triangular geometry of (111) surfaces can cause a further tilting of the anisotropy axis.

[32] P. Gambardella, S. Rusponi, M. Veronese, S.S. Dhesi, C. Grazioli, A. Dallmeyer, I. Cabria, R. Zeller, P. H. Dederichs, K. Kern, C. Carbone, and H. Brune, Science 300, 1130 (2003).

[33] S. Rusponi, T. Cren, N. Weiss, M. Epple, P. Buluschek, L. Claude, and H. Brune, Nature Materials 2, 546 (2003).

[34] A.B. Shick, F. Máca, and P.M. Oppeneer, Phys. Rev. B 69, 212410 (2004).

[35] S. Baud, G. Bihlmayer, S. Blügel, and Ch. Ramseyer, Surf. Sci. 600, 4301 (2006).

[36] S. Baud, Ch. Ramseyer, G. Bihlmayer and S. Blügel, Phys. Rev. B. 73, 104427 (2006).

[37] A. Kukunin, J. Prokop, and H. J. Elmers, Phys. Rev. B 76, 134414 (2007).

[38] Y. Mokrousov, G. Bihlmayer, S. Heinze, and S. Blügel, Phys. Rev. Lett. 96, 147201 (2006).

[39] R. Cordery, S. Sarker, and J. Tobochnik, Phys. Rev. B 24, 5402(R) (1981).

[40] A. Vindigni, A. Rettori, L. Bogani, A. Caneschi, D. Gatteschi, R. Sessoli, and M.A. Novak, Appl. Phys. Lett. 87, 073102 (2005).

[41] C. Serpico, I.D. Mayergoyz, and G. Betrotti, J. Appl. Phys. 93, 6909 (2003).

[42] O. Šipr, S. Bornemann, J. Minár, S. Polesya, V. Popescu, A. Šimunek, and H. Ebert, J. Phys.: Condens. Matter 19, 096203 (2006).

[43] J. Minar, S. Bornemann, O. Šipr, S. Polesya, and H. Ebert, Appl. Phys. A 82, 139 (2006); O. Šipr, private communication.

[44] A.B. Klautau and S. Frota-Pessoa, Int. J. Modern Phys. B 20, 5281 (2006).

[45] A. M. Conte, S. Fabris, and S. Baroni, Phys. Rev. B 78, 014416 (2008).

[46] G. Nahrwold, J.M. Scholtyssek, S. Motl-Ziegler, O. Albrecht, U. Merkt, and G. Meier, J. App. Phys. 108, 013907 (2010).

[47] J. Kötzler, D. Görlitz, and F. Wiekhorst, Phys. Rev. B 76, 104404 (2007).

[48] S. Krause, L. Berbil-Bautista, G. Herzog, M. Bode, and R. Wiesendanger, Science 317, 1537 (2007).

[49] Very recently, new developments in pump-probe scanning tunneling spectroscopy have made possible the access of spin excitations in the nanosecond regime by this method. See S. Loth, M. Etzkorn, C.P. Lutz, D.M. Eigler, and A.J. Heinrich, Science 329, 1628 (2010). 
[50] G.N. Milstein and M.V. Tretyakov, Siam J. Numer. Anal. 34, 2142 (1997). 